\title{
Hookworm infection and associated risk factors among clinically confirmed podoconiosis patients and healthy residents in Debre Elias district, East Gojjam Zone
}

Abebe Alemu

Injibara General hospital

Habtie Tesfa

University of Gondar

Ayalew Jejaw Zeleke

University of Gondar

Yalewayker Tegegne

University of Gondar

Yibeltal Aschale

University of Gondar

Ayenew Addisu

University of Gondar

Ligabaw Worku ( $\square$ ligabaw@gmail.com )

University of Gondar College of Medicine and Health Sciences

Research article

Keywords: Hookworm, Podoconiosis, risk factors, East Gojam

Posted Date: August 21st, 2019

DOI: https://doi.org/10.21203/rs.2.13272/v1

License: (c) (1) This work is licensed under a Creative Commons Attribution 4.0 International License.

Read Full License 


\section{Abstract}

\section{Background}

Hookworm is a major public health problem in Ethiopia. The prevalence of Hookworm and associated factors among podoconiosis patients and healthy residents has not been studied.Objective to determine the prevalence of Hookworm and associated risk factors among podoconiosis patients and healthy residents.

\section{Methods}

A cross-sectional study was conducted at Debre Elias from January to February 2018. One hundred ninety two participants were included. Stool sample was collected for microscopy, kato-katz and formalin ether concentration technique. Logistic regressions were performed to assess association between variables. P-value $<0.05$ was used as statistically significant.

Result

The prevalence of Hookworm among podoconiosis patients was $84.4 \%$ and $75 \%$ among control. Hookworm infection was relatively higher among podoconiosis patients $52.9 \%$. Higher age group, illiterate and farmers more affected with high proportion of podoconiosis patients. Being a formers, no history of stool examination and no shoe wearing habit were significantly associated with hookworm infection $(\mathrm{AOR}=4.1 ; 95 \% \mathrm{Cl}=(1.65-10.11)),(\mathrm{AOR}=3.03 ; 95 \% \mathrm{Cl}=(1.11-8.25))$ and $(\mathrm{AOR}=3.9 ; 95 \% \mathrm{Cl}=$ (1.11-14.24) respectively.

\section{Background}

Hookworms are intestinal parasites of humans transmitted through contact with the infective larvae found in focally polluted soil. Anslostoma duodenale and Necater americanus are the common hookworm species. Humans become infected through skin penetration by infective larvae in the soil [13]. It mainly affects adults than preschool and school aged children. Infected people mostly live in poor conditions in developing nations, where households lack sufficient waste disposal facilities and latrine [4, $5]$.

Hookworm are a major public health burden throughout many countries of the world where access to anthelmintics is limited [6]. Globally, 5.1 billion people are thought to be at risk of acquiring hookworm infection, of which approximately 500 million people are currently affected [7]. According to the recent WHO global health estimates, hookworm diseases account for over 1.7 million disability-adjusted life years (DALYs) [8].

Prevalence of hookworm infection is also higher among podoconiosis patients in the highland of Ethiopia and contribute for malnutrition and iron deficiency anemia $[9,10]$. 
Podoconiosis is a non-filarial elephantiasis of the lower leg that affects barefoot subsistence farmers exposed to fine mineral particles that originate from red clay volcanic soil in highland tropical areas [11]. Hookworm and podoconiosis are highly prevalent in tropical and sub-tropical areas of developing countries and have geographical overlapping distribution due to the presence of many bare footed farmers who are actively working on agricultural activities [10,12]. Similarly lack of safe water supply, latrine and appropriate waste disposal system contribute for the highest infestation of larvae of hookworm $[12,13]$. Poor personal hygiene, farming on infested soil, absence of shoe wear habit, and inadequate or absent of sanitation practice expose people for hookworm in tropical and subtropical regions and make hookworm common in developing countries $[10,14]$.

Podoconiosis is an immune disease affecting the lymph vessels of the lower legs and create an opportunity for hookworm infection. $[14,15,16]$. Thus the assessment of hookworm among podoconiosis patient and healthy control is very important for the management of hookworm, podoconiosis and co infected patients.

The prevalence of hookworm and podoconiosis has been studied previously in different locality in Ethiopia; however evidences are not enough concerning the extent of hookworm on podoconiosis patient and coexistence in most of the country. Therefore, this study was aim to determine the prevalence of Hookworm infection and associated risk factors among clinically confirmed podoconiosis patients and healthy residents.

\section{Materials And Methods}

\section{Study design, period and area}

A community based comparative cross-sectional study was conducted in Debre Elias district, East Gojjam Zone from January 11 to February 7, 2018. According to 2017 Debre Elias district communication office data; Debre Elias is located $340 \mathrm{~km}$ north of Addis Ababa. Ninety one percent of the district was within the altitude of $2100-2300$ meters while $9 \%$ were $1500-2100$ meters above sea level. A total population of the town is about 100,797 of whom 49,955 were males and 50,842 were females. The district has 12 , 970 urban and 87,827 rural populations.

\section{Study design}

A community based comparative cross-sectional study was conducted to determine prevalence of hook worm infection and associated risk factors among clinically confirmed podoconiosis patient and healthy residence in Debre Elias district east.

\section{Study population and sample size}


The sample size was determined using the two population proportion formula, $\left(n=\left(Z_{\alpha / 2}+Z_{\beta}\right)^{2 *}\left(p_{1}\left(1-p_{1}\right)\right.\right.$ $\left.\left.+p_{2}\left(1-p_{2}\right)\right) /\left(p_{1}-p_{2}\right)^{2}\right)$ considering $95 \%$ level of confidence interval, $80 \%$ power of the study and $47.6 \%$ and $33.1 \%$ prevalence podoconiosis and healthy residence which resulted in the sample size of 175 . By adding a 10\% contingency, we recruited 192 (96 podoconiosis patients and 96 neighborhood residents) in the study. A two stage cluster sampling technique was used to select study participants and podoconiosis patients were proportionally allocated to each of the selected cluster. A systematic random sampling technique was used to select podoconiosis patients taking the list of each cluster victim's registration book as a sampling frame. For each podoconiosis study participants their healthy residents were included.

\section{Data Collection}

\section{Socio- demographic Data Collection.}

Socio- demography and possible associated risk factors were assessed using a pretested and standardized questionnaire by health officers through interview, which was translated into the local language, Amharic

\section{Stool collection and parasitic examination:}

Each study participants was advised to bring about $5 \mathrm{gm}$ of fresh stool and was given a labeled, clean, dry, and leak proof stool cup. Direct saline and Kato thick smear were processed inside the temporary laboratory established at the site. Formalin preserved stool sample was transported into Debre Elias health center and processed using formol-ether concentration technique.

\section{Quality control}

All the necessary reagents, chemicals, and the performance of kits were checked by known positive and negative samples before processing and examination of samples of the study subjects. The data was checked for completeness and any incomplete or misfiled questionnaires were re-corrected under supervision. All slides were examined twice by two microscopists independently. The result of laboratory examination was recorded on well-prepared format carefully for confirmation of the result.

\section{Data analysis and interpretation}

Data were entered and analyzed using SPSS version 20 software. The frequency distribution of both dependent and independent variables was worked out. Binary and multivariate logistic regressions were used to assess any significant association between independent and dependent variables. P-Values less than 0.05 were taken as statistically significant. 


\section{Results}

\section{Socio-demographic characteristics of study participants}

A total of 192 study subjects (96 podoconiosis patients and 96 healthy residents) were participated. From all study participants, 46/96 (48\%) were females and 50/96 (52\%) were males in both podoconiosis patients and healthy residents respectively. Of the total 192 study subjects 60 (31.3\%) were 55 years old and above, 97 (50.5\%) were illiterate and 127 (66.1\%) were farmers(Table 1).

\section{Prevalence of Hookworm and other helminthes}

The prevalence of intestinal helminthes among podoconiosis patients were 86/92 (89.6\%) whereas $75 / 92$ (78.1\%) were healthy residence with the overall prevalence of 161/192 (83.9\%). Hookworm, S.stercolaris and E.vermicularis were identified with the prevalence rate of $79.7 \%, 3.6 \%$ and $0.5 \%$ respectively. Hookworm was the predominant parasite in both podoconiosis patients and healthy residents with the prevalence rate of $84.4 \%$ and $75 \%$. Strongloides stercolaris were identified $5.2 \%$ and $2.1 \%$ among podoconiosis patients and healthy residents. Multiple infections of Hookworm and S.stercolaris were observed in both podoconiosis patients (4.2\%) and healthy residents (2.1\%) (Table 2).

\section{The proportion of Hookworm infection among podoconiosis patients and healthy residents}

Hookworm infection was relatively higher among podoconiosis patients $81 / 153(52.9 \%)$ than control $72 / 153$ (47.1\%). Podoconiosis patients age ranged from 35- 54 was more infected than healthy controls. Both sexes of podoconiosis patients were highly infected by hookworm. Seventy nine percent of illiterate study subjects were infected with the proportion of $60.8 \%$ podoconiosis patients and $39.2 \%$ healthy control. Farmers was more affected groups $110 / 153$ (71.9\%) among this $56.4 \%$ and $43.6 \%$ was podoconiosis patients and healthy control respectively (Table 3 ).

\section{Univariate and Multivariate Risk Factor Analysis for hookworm}

The relationship between socio-demographic variables and infection with hookworm was analyzed using univariate and multivariate logistic regression. Being a formers was about four times more likely to have hookworm than others $(\mathrm{AOR}=4.1 ; 95 \% \mathrm{Cl}=(1.65-10.11))$. The report shows that having no previous history of stool examination and no shoe wearing habit were significantly associated with hookworm infection with $(\mathrm{AOR}=3.03 ; 95 \% \mathrm{Cl}=(1.11-8.25))$ and $(\mathrm{AOR}=3.9 ; 95 \% \mathrm{Cl}=(1.11-14.24))$ respectively (Table 4).

\section{Discussion}


Hookworm and podoconiosis are neglected tropical diseases which have been given relatively little attention by national government and international organization. Hookworm infection is high in coendemic area. However, hookworm infection can cause gastrointestinal bleeding, iron deficiency anemia, hypoproteinemia, zinc deficiencies and malnutrition $[1,10,11]$.

In the present study, the overall average prevalence of intestinal helminthes was $83.9 \%$, which was higher when compared with the studies conducted in different parts of Ethiopia, such as Wolaita[10], Hawassa [17], Jimma [18] and other countries Daressalaam urban Tanzania [19], Kenya (0.2\%) [20], and Nepal (9.1\%) [21].These differences in prevalence could be due to the use of different study subject, diagnostic methods and sociodemographic difference in the study subjects or due to a reflection of the local endemicity and geographic condition of the study area.

Prevalence of intestinal helminthes was higher among clinically confirmed podoconiosis patients as compared with healthy residents in the present study which was similar to Wolaita Zone, Southern Ethiopia. This might be due to the difference in skin texture and size of the foot which help the infective larva to easily access and penetrate in podoconiosis patients. Similarly the prevalence of intestinal helminthes among healthy participants was greater than the study conducted in Wolaita (33.1\%) [10],Hawassa (48.8\%) [17], Jimma (46.63\%) [18], Daressalaam urban Tanzania (25.9\%) [19], Kenya (0.2\%) [20], and Nepal (9.1\%) [21]. The difference might be due to geographical and socioeconomic variation because different areas may have difference in sanitation practice, warm and moist soils which facilitate the transmission and development of parasite ova and larva.

The results presented here relatively higher prevalence of hookworm among podoconiosis patients than controls, which was higher than a study conducted in Wolaita Zone [10]. This might be due to geographical and sampling period difference because including Jun, a rainy season, as the study time might decrease the soil temperature important for hookworm larva development in Wolaita.

Regarding to the age groups, 35-44 and 54-54 years podoconiosis patient were highly affected groups, with a prevalence rate of $60 \%$ and $57.9 \%$ respectively. This prevalence difference might be due to long exposure rate of irritant clay soil.

In this study different factors had association with the increase prevalence of hookworm after multivariate analysis. This study revealed that absence of shoe wearing habit was significantly associated with hookworm infection $(\mathrm{AOR}=3.9 ; 95 \% \mathrm{Cl}=(1.11-14.24)$ This study finding was supported by a study conducted in Wolaita Zone Southern Ethiopia[10], and Orang Asli in Peninsular Malaysia[22]. This factor is an important evidence for the high prevalence of hookworm because the transmission of the parasite is through penetration of skin by the infective filariform larvae and prevented by reduction of skin contact with potentially infected soil by wearing shoes[1].

Prevalence of hookworm also significantly associated with farmers (AOR $=4.1 ; 95 \% \mathrm{Cl}=(1.65-10.11)$ ). This was the same to the study conducted in Wolaita Zone [10]. This might be due to farmer's exposure for more constant contact of contaminated soil. In addition farmers engaged in agricultural activities 
where shoe wearing is not convenient and no habit of getting lunch with water for hand washing in the work environment.

Absence of previous stool examination for hookworm infection was a significant risk factor identified (AOR $=3.03 ; 95 \% \mathrm{Cl}=(1.11-8.25)$ This might indicate having stool examination history is preventive because a diagnosed individual would take antihelmenthic medication in positive finding and decrease exposure history to parasites because treatment of hookworm is an important measure to decrease their prevalence [23].

\section{Limitations}

The study was not assessed the detailed exposure history of hookworm, host immunity and nutrition status of the study subject which was a potential confounder for risk factor assessment.

\section{Declarations}

\section{Ethical approval and consent to participate}

Permission to conduct the study was obtained from Research and Ethics Committee of the School of Biomedical and Laboratory Sciences, University of Gondar (reference no. SBMLS/872/10), and Debre Elias district health office. Informed verbal consent was also obtained from each study participants. Participants who were positive for intestinal parasite(s) were communicated to responsible clinicians and treated with appropriate drugs for free.

\section{Availability of data and material}

I confirmed that all the data for this manuscript are available, if someone wants to request the data can contact all authors.

\section{Funding}

Financial support was provided the University of Gondar. However the university has no role in the design of the study, collection of data, analysis, and interpretation of the result and in writing of the manuscript.

\section{Acknowledgments}

The authors would like to thank the University of Gondar for financial support and Debre Elias health center for providing excellent laboratory facilities for processing the stool samples. They also gratefully thank the staff members of Debre Elias Health Center for treating the infected study participants, 
community volunteer, health service providers of podoconiosis victims association. Lastly, our thanks go to all study participants for their consent.

\section{Consent for publication}

Not applicable.

\section{Competing of Interests}

All authors declare that they have no conflict of interests in relation to their work

\section{Authors' Contribution}

Abebe Alemu conceived the study, undertook statistical analysis, and drafted the initial and final paper. Ligabaw Worku, Ayenew Addisu, Yibeltal Aschale initiated the study, made major contributions to the study design, reviewed the initial and final drafts of paper, and conducted data analysis. Ayalew Jejaw, Habtie Tesfa, and Yalewayker Tegegne participated in sample collection and the performance of laboratory diagnosis. All authors contributed to the writing of the paper and approved the submitted version of the paper.

\section{References}

1. Knopp S, Steinmann P, Keiser J, Utzinger J: Nematode infections: soil-transmitted helminths and Trichinella. Infectious Disease Clinics 2012, 26(2):341-358.

2. Bethony J, Brooker S, Albonico M, Geiger SM, Loukas A, Diemert D, Hotez PJ: Soil-transmitted helminth infections: ascariasis, trichuriasis, and hookworm. The Lancet 2006, 367(9521):15211532.

3. Oswald WE, Stewart AE, Kramer MR, Endeshaw T, Zerihun M, Melak B, Sata E, Gessese D, Teferi T, Tadesse Z: Association of community sanitation usage with soil-transmitted helminth infections among school-aged children in Amhara Region, Ethiopia. Parasites \& vectors 2017, 10(1):91.

4. Gabrie JA, Rueda MM, Canales M, Gyorkos TW, Sanchez AL: School hygiene and deworming are key protective factors for reduced transmission of soil-transmitted helminths among schoolchildren in Honduras. Parasites \& vectors 2014, 7(1):354.

5. Bitew AA, Abera B, Seyoum W, Endale B, Kiber T, Goshu G, Admass A: Soil-transmitted helminths and Schistosoma mansoni infections in Ethiopian Orthodox Church students around lake tana, northwest Ethiopia. PloS one 2016, 11(5):e0155915.

6. Malik S: Gastrointestinal nematodes and their management: a review. Environment Conservation Journal 2015, 16(3):95-103. 
7. Bartsch, S. M.; Hotez, P. J.; Asti, L.; Zapf, K. M.; Bottazzi, M. E.; Diemert, D. J.; Lee, B. Y. The global economic and health burden of human hookworm infection. PLoS Negl. Trop. Dis. 2016, 10, e0004922.

8. World Health Organization. Global Health Estimates Summary Tables. DALYs by Cause, Age and Sex, by WHO Region, 2000-2015; WHO: Geneva, Switzerland, 2016.

9. Hotez PJ, Brooker S, Bethony JM, Bottazzi ME, Loukas A, Xiao S: Hookworm infection. New England Journal of Medicine 2004, 351(8):799-807.

10. Taye B, Alemayehu B, Birhanu A, Desta K, Addisu S, Petros B, Davey G, Tsegaye A: Podoconiosis and soil-transmitted helminths (STHs): double burden of neglected tropical diseases in Wolaita zone, rural Southern Ethiopia. PLoS neglected tropical diseases 2013, 7(3):e2128.

11. Molla YB, Tomczyk S, Amberbir T, Tamiru A, Davey G: Patients' perceptions of podoconiosis causes, prevention and consequences in East and West Gojam, Northern Ethiopia. BMC Public Health 2012, 12:828.

12. Amenu D: Health impact of intestinal helminth infections among podoconiosis patients. Trends in Bacteriology 2014, 1(1):2.

13. Ekpo UF, Odoemene SN, Mafiana CF, Sam-Wobo SO: Helminthiasis and hygiene conditions of schools in Ikenne, Ogun State, Nigeria. PLoS Neglected Tropical Diseases 2008, 2(1):e146.

14. Strunz EC, Addiss DG, Stocks ME, Ogden S, Utzinger Jr, Freeman MC: Water, sanitation, hygiene, and soil-transmitted helminth infection: a systematic review and meta-analysis. PLoS medicine 2014, 11(3):e1001620.

15. Yimer M, Hailu T, Mulu W, Abera B: Epidemiology of elephantiasis with special emphasis on podoconiosis in Ethiopia: a literature review. Journal of vector borne dis 2015, 52(2):111.

16. Davey G, Tekola F, Newport MJ: Podoconiosis: non-infectious geochemical elephantiasis. Transactions of the Royal Society of Tropical Medicine and Hygiene 2007, 101(12):1175-1180.

17. Assefa S, Erko B, Medhin G, Assefa Z, Shimelis T: Intestinal parasitic infections in relation to HIV/AIDS status, diarrhea and CD4 T-cell count. BMC infectious diseases 2009, 9(1):155.

18. Mengistu A, Gebre-Selassie S, Kassa T: Prevalence of intestinal parasitic infections among urban dwellers in southwest Ethiopia. Ethiopian Journal of Health Development 2007, 21(1):12-17.

19. Mhimbira F, Hella J, Said K, Kamwela L, Sasamalo M, Maroa T, Chiryamkubi M, Mhalu G, Schindler C, Reither K: Prevalence and clinical relevance of helminth co-infections among tuberculosis patients in urban Tanzania. PLoS neglected tropical diseases 2017, 11(2):e0005342.

20. Masaku J, Mutungi F, Gichuki PM, Okoyo C, Njomo DW, Njenga SM: High prevalence of helminths infection and associated risk factors among adults living in a rural setting, central Kenya: a crosssectional study. Tropical medicine and health 2017, 45(1):15.

21. Parajuli R, Fujiwara T, Umezaki M, Konishi S, Takane E, Maharjan M, Tachibana K, Jiang H, Pahari K, Watanabe C: Prevalence and risk factors of soil-transmitted helminth infection in Nepal.

Transactions of The Royal Society of Tropical Medicine and Hygiene 2014, 108(4):228-236. 
22. Ngui R, Aziz S, Chua KH, Aidil RM, Lee SC, Tan TK, Sani MM, Arine AF, Rohela M, Lim YA: Patterns and risk factors of soil-transmitted Helminthiasis among Orang Asli subgroups in Peninsular Malaysia. The American journal of tropical medicine and hygiene 2015, 93(2):361-370.

23. Jad B, Dogra S, Mahajan B: Significant decrease in prevalence of intestinal parasites among patients seeking treatment in a tertiary care hospital in Jammu: a changing trend. Int J Curr Microbiol App Sci 2015, 4:659-664. 